\title{
THE WORLD OF QUANTUM NOISE AND THE FUNDAMENTAL OUTPUT PROCESS
}

\author{
V. P. BELAVKIN, O. HIROTA, AND R. HUDSON
}

\begin{abstract}
A stationary theory of quantum stochastic processes of second order is outlined. It includes KMS processes in wide sense like the equilibrium finite temperature quantum noise given by the Planck's spectral formula. It is shown that for each stationary noise there exists a natural output process which is identical to the noise in the infinite temperature limit, and flipping with the noise if the time is reversed at finite temperature. A canonical Hilbert space representation of the quantum noise and the fundamental output process is established and a decomposition of their spectra is found. A brief explanation of quantum stochastic integration with respect to the input-output processes is given using only correlation functions. This provides a mathematical foundation for linear stationary filtering transformations of quantum stochastic processes. It is proved that the colored quantum stationary noise and its time-reversed version can be obtained in the second order theory by a linear nonadapted filtering of the standard vacuum noise uniquely defined by the canonical creation and annihilation operators on the spectrum of the input-output pair.
\end{abstract}

\section{INTRODUCTION}

In this paper we develop a correlation theory of stationary quantum noise, give its spectral analysis and classification and extend the theory of quantum stochastic integration [1] to colored quantum noise [2]. The typical example of such noise is given by the Planck spectral formula. It shows that finite temperature equilibrium quantum noise is not white in contrast to classical equilibrium noise given by the Nyquist spectral formula. The weak coupling limit and rotating wave approximation [2, 3] make it possible to approximate the equilibrium quantum noise by canonical pairs of noncommutative white noises in a narrow spectral band near a resonant frequency.

Although such an approximation is sufficient in quantum optics and in many other practical cases, it is not satisfactory from a purely theoretical point of view because it does not correctly predict the simplest quantum stochastic motion of a free Brownian particle in an equilibrium environment, when the resonant frequency is zero.

Date: September 26, 1994.

Key words and phrases. Quantum Noise, Output Process, Quantum Correlations, Time Reversion, Spectral Analysis, Stochastic Integration, Linear Filtering.

The first author thanks to Matt James for support and hospitality in ANU, Canberra, where this paper was prepared for the current publication .

This paper was originally published in: Proceedings of the 2nd QCMC conference: Quantum Communications and Measurement, 3-19, Plenum Press, New-York and London 1995. 
In order to describe the output stochastic processes of quantum filters and other devices of quantum measurement and communications as functional transformations of the equilibrium noise, we also need a generalization of the notion of quantum output fields , 4, 5, 6] in the framework of stochastic integration with respect to the colored quantum noise

The well developed quantum stochastic calculus and noncommutative extension of Itô integration to vacuum noise with canonical commutation relations in the time domain [6, 7] is not directly applicable to such cases, neither is the calculus and integration with respect to stochastic models of temperature quantum noise with flat spectrum [5, 8].

But we can use the methods of quantum stochastic integration in the spectral domain, in which the stationary quantum noise is $\delta$-correlated with a certain modification. The latter is required because the colored quantum processes are not frequency-stationary and need not be adapted in the spectral representation. Although non-stationary and non-adapted theories of quantum stochastic calculus and integration have been already established [9, 10, 11, here we will use the much more simple approach of mean square integration which is sufficient for the nonadapted linear transformations. The corresponding classical theory of stochastic integration was developed by Wiener before the Itô integration theory and is based on the possibility of representing any (colored) process stationary in the wide sense as a linear filtering integration of the standard white noise.

Here we shall prove that any quantum process stationary in the wide sense can be also obtained from a standard one also by filtering. In fact, we will show that it can be obtained by mean square integration with respect to a canonical pair of orthogonal quantum integrators describing the standard zero temperature (vacuum) noise in the second order. Although such a possibility is known for the Gaussian case as a Bogolubov transformation which doubles degrees of freedom of the noise in such representation, it has not been realized in the theory of quantum noise and integration.

We will remove this unpleasant feature by deriving the fundamental output process for a given quantum noise as the canonically time-reversed noise, which commutes with the input noise and has maximal possible correlations with it. In the classical (or infinite temperature) case, the fundamental output process coincides with the noise. In the quantum case it gives the best possible nondemolition filtering and time continuous indirect observation of the noise.

The fundamental output process for a given Gaussian quantum noise in the framework of quantum stochastic calculus was introduced in [12] and the possibility of its nondemolition observation was demonstrated for Markovian models of interaction with a quantum open system in the quantum theory of filtering (see the recent survey 13 and papers cited therein).

To explain the idea of the fundamental output process, let us represent a canonical Bose-noise annihilation and creation pair $\mathrm{b}, \mathrm{b}^{\dagger}$ with non-zero temperature correlations

$$
\left\langle\mathrm{b}^{\dagger} \mathrm{b}\right\rangle=n, \quad\left\langle\mathrm{bb}^{\dagger}\right\rangle=n+1
$$

by the linear combination $\mathrm{b}=\sqrt{n+1} \mathrm{a}+\sqrt{n} \mathrm{c}$ of the zero temperature pairs $\mathrm{a}, \mathrm{a}^{\dagger}$ and $\mathrm{c}, \mathrm{c}^{\dagger}$ with 


$$
\left[\mathrm{a}, \mathrm{a}^{\dagger}\right]=1, \quad\left[\mathrm{c}^{\dagger}, \mathrm{c}\right]=1, \quad\left[\mathrm{a}^{\dagger}, \mathrm{c}\right]=[\mathrm{a}, \mathrm{c}]=0 .
$$

Then the combination $\tilde{b}=\sqrt{n} \mathrm{a}+\sqrt{n+1} \mathrm{c}$ defines a canonical pair $\tilde{b}, \tilde{b}^{\dagger}$ of output creation and annihilation operators with correlations

$$
\left\langle\tilde{\mathrm{b}}^{\dagger} \tilde{\mathrm{b}}\right\rangle=n+1,\left\langle\tilde{\mathrm{b}} \tilde{\mathrm{b}}^{\dagger}\right\rangle=n
$$

which commutes with the noise, $[\tilde{\mathrm{b}}, \mathrm{b}]=\left[\tilde{\mathrm{b}}, \mathrm{b}^{\dagger}\right]=0$, and provides the maximal mutual correlations $\left\langle\tilde{\mathrm{b}} \mathrm{b}^{\dagger}\right\rangle=\sqrt{n(n+1)}$, corresponding to correlation coefficient $r=1$.

In the classical case, the latter would mean that $\tilde{b}=b$, but this is not the case: $\tilde{b}$ commutes with $b^{\dagger}$ but $b$ does not. Nevertheless $\tilde{b}$ is uniquely defined for the noise $\mathrm{b}$ by the described properties as the time-reversed noise, which in the real arrow of time plays the role of the fundamental output process. Moreover, the zero temperature pair a, $c$ is uniquely determined by the pair $(b, \tilde{b})$, being given by

$$
\mathrm{a}=\sqrt{n+1} \mathrm{~b}-\sqrt{n} \tilde{\mathrm{b}}, \quad \mathrm{c}=\sqrt{n+1} \tilde{\mathrm{b}}-\sqrt{n} \mathrm{~b} .
$$

\section{Quantum Correlations and Reversed Processes}

Here we sketch of a second order theory of quantum noise and linear filtering of stationary signals, initiated more than 20 years ago in the pioneer paper [14]. A real scalar quantum stochastic noise as a process $\mathrm{x} .=\left(\mathrm{x}_{j}\right)$ in second order is completely determined by the zero mean values $\left\langle\mathrm{x}_{j}\right\rangle=0$ and finite variances alone $\left\langle\mathrm{x}_{j}^{2}\right\rangle<\infty$ of the Hermitian variables $\mathrm{x}_{j}=\mathrm{x}_{j}^{\dagger}$ and also by their not necessarily symmetric covariances, or correlations $\left\langle\mathrm{x}_{i} \mathrm{x}_{j}\right\rangle \neq\left\langle\mathrm{x}_{j} \mathrm{x}_{i}\right\rangle$. The products $\mathrm{x}_{i} \mathrm{x}_{j}$ in $\langle\cdot\rangle$ form a Hermitian-positive kernels $\left\langle\mathrm{x}_{i} \mid \mathrm{x}_{j}\right\rangle$ which define the scalar products in a complex Euclidean space. By writing an invertible correlation kernel $\left\langle\mathrm{x}_{i} \mathrm{x}_{j}\right\rangle \equiv$ $\left\langle\mathrm{x}_{i} \mid \mathrm{x}_{j}\right\rangle$ as a bra-ket inner product $\left\langle\mathrm{x}_{i}|\cdot| \mathrm{x}_{j}\right\rangle$, we can describe the quantum noise in second order by real vectors $\left|\mathrm{x}_{j}\right\rangle=\left|\mathrm{x}_{j}\right\rangle^{\sharp}$ equipped with the involution $|\mathrm{z}\rangle \mapsto|\mathrm{z}\rangle^{\sharp}=$ $\left|z^{\dagger}\right\rangle$. The latter, defined by

$$
\left|\sum \zeta_{j} \mathrm{x}_{j}\right\rangle^{\sharp}=\sum \zeta_{j}^{*}\left|\mathrm{x}_{j}\right\rangle \quad \forall \zeta_{j} \in \mathbf{C}
$$

such that $|z\rangle^{\sharp \sharp}=|z\rangle$, gives vector representation of the Hermitian conjugation $\mathrm{z} \mapsto \mathrm{z}^{\dagger}=\sum \zeta_{j}^{*} \mathrm{x}_{j}$ with $\zeta_{j}^{*}=\overline{\zeta_{j}}$ for the complex combinations $\mathrm{z}=\sum \zeta_{j} \mathrm{x}_{j} \equiv \zeta \cdot \mathrm{x}$ of the Hermitian variables $\mathrm{x}_{j}$ given by raws $\zeta .=\left(\zeta_{i}\right)$.

Let us interpret the index $j$ as "discrete time", e.g. $j \in \mathbf{Z}$ when $\mathrm{x} .=\left(\mathrm{x}_{j}\right)_{j \in \mathbf{Z}}$ is two sided infinite sequence $\left(\cdots, \mathrm{x}_{-1}, \mathrm{x}_{0}, \mathrm{x}_{1}, \cdots\right)$ of Hermitian variables with the time ordered correlations

$$
K_{i j}=\left\langle\mathrm{x}_{i} \mathrm{x}_{j}\right\rangle \equiv\left\langle\mathrm{x}_{i} \mid \mathrm{x}_{j}\right\rangle, \quad i>j \in \mathbf{Z}
$$

and the reverse ordered $K_{j i}=\left\langle\mathrm{x}_{j} \mid \mathrm{x}_{i}\right\rangle$ which we need to define the scalar product of the corresponding vectors $\left|\mathrm{x}_{j}\right\rangle$. In contrary to the classical theory, these correlations need not be real valued even for Hermitian $\mathrm{x}_{j}$, so that time reversal corresponds to their complex conjugation:

$$
K_{j i}=\left\langle\mathrm{x}_{j} \mathrm{x}_{i}\right\rangle=\overline{\left\langle\mathrm{x}_{i} \mathrm{x}_{j}\right\rangle}=\bar{K}_{i j} .
$$


Although the reversed correlations may be considered as not "observable" in the real arrow of time, they are defined by complex conjugation in such a way that the Hermitian matrix $K=\left[K_{i j}\right]$ is positive definite:

$$
\zeta^{*} K \cdot \zeta:=\sum \zeta_{i}^{*}\left\langle\mathrm{x}_{i} \mathrm{x}_{j}\right\rangle \zeta_{j}=\left\langle\mathrm{z}^{\dagger} \mathrm{z}\right\rangle \geq 0
$$

as is the complex conjugate $=$ transpose $\bar{K}=\left[\bar{K}_{i j}\right]=\left[K_{j i}\right]=\widetilde{K}$ :

$$
\zeta^{*} \widetilde{K} \cdot \zeta:=\sum \zeta_{j}\left\langle\mathrm{x}_{j} \mathrm{x}_{i}\right\rangle \zeta_{i}^{*}=\left\langle\mathrm{z} \mathrm{z}^{\dagger}\right\rangle \geq 0 .
$$

We claim that $\bar{K}$ can be treated as the correlation matrix

$$
\widetilde{K}_{i j}=\left\langle\widetilde{\mathrm{x}}_{i} \widetilde{\mathrm{x}}_{j}\right\rangle \equiv\left\langle\widetilde{\mathrm{x}}_{i} \mid \widetilde{\mathrm{x}}_{j}\right\rangle, \quad \forall i \geq j \in \mathbf{Z}
$$

of another sequence $\widetilde{\mathrm{x}}_{i}=\left(\cdots, \widetilde{\mathrm{x}}_{-1}, \widetilde{\mathrm{x}}_{0}, \widetilde{\mathrm{x}}_{1}, \cdots\right)$ of Hermitian $\widetilde{\mathrm{x}}_{i}$ commuting with $\mathrm{x}_{j}$ for all $j \in \mathbf{Z}$ which is maximally correlated to $\mathbf{x} .=\left(\mathrm{x}_{j}\right)_{j \in \mathbf{Z}}$ in the sense that the real covariance matrix $G=\left[\left\langle\widetilde{\mathrm{x}}_{i} \mathrm{x}_{j}\right\rangle\right]$ ispositive and symmetric as the geometric mean $G=(K \cdot \widetilde{K})^{1 / 2}$ of $K$ and $\widetilde{K}=\bar{K}$. The sequence $\widetilde{\mathrm{x}}$ describes the fundamental output process as reversed noise by the quantum Hermitian variables $\widetilde{\mathrm{x}}_{j}=\widetilde{\mathrm{x}}_{j}^{\dagger}$ which will also be represented by real vectors $\left|\widetilde{\mathrm{x}}_{j}\right\rangle=\left|\widetilde{\mathrm{x}}_{j}\right\rangle^{b}$ with respect to another involution $|\widetilde{\mathrm{z}}\rangle \mapsto|\widetilde{\mathrm{z}}\rangle^{b}$ uniquely defined on the complex combination $\widetilde{\mathrm{z}}=\zeta \cdot \widetilde{\mathrm{x}}$ by complex conjugation of the coefficients $\zeta_{j} \in \mathbf{C}$. This suggests a natural norm

$$
\|\zeta\|^{2}=\left\langle\mathrm{z}^{\dagger} \mathrm{z}\right\rangle+\left\langle\mathrm{z} \quad \mathrm{z}^{\dagger}\right\rangle=\zeta^{*}(K+\widetilde{K}) \cdot \zeta
$$

in the space $\mathcal{E}$ of test sequences $\zeta=\left(\zeta_{i}\right)$ for the simultaneous treatment of linear combinations $|\mathrm{z}\rangle=\sum \zeta_{j}\left|\mathrm{x}_{j}\right\rangle$ of the $\sharp$ - real vectors $\left|\mathrm{x}_{j}\right\rangle=\left|\mathrm{x}_{j}\right\rangle^{\sharp}$, and the combinations $|\widetilde{\mathrm{z}}\rangle=\sum \zeta_{j}\left|\widetilde{\mathrm{x}}_{j}\right\rangle$ of $b$ - real vectors $\left|\widetilde{\mathrm{x}}_{j}\right\rangle=\left|\widetilde{\mathrm{x}}_{j}\right\rangle^{b}$, representing $\widetilde{\mathrm{x}}_{j}=\widetilde{\mathrm{x}}_{j}^{\dagger}$.

From now on for the sake of simplicity we shall restrict ourselves to the case when the matrices $K$ and $\widetilde{K}$ commute as it is in the stationary case

$$
K_{i j}=k_{i-j}, \quad k_{-j}=\bar{k}_{j}, \quad \forall j \in \mathbf{Z}
$$

(see also 12 for a more general, noncommutative case.) In this case that covariances of $\mathrm{x}_{i}$ and $\widetilde{\mathrm{x}}_{j}$ are described by the real symmetric matrix

$$
G=(K \bar{K})^{1 / 2}=(\bar{K} K)^{1 / 2}=\bar{G}
$$

as geometric mean of the commuting $K$ and $\bar{K}$, corresponding to maximal correlations with zero commutators between $\mathrm{x}$ and $\widetilde{\mathrm{x}}$ :

$$
G_{i j}=\left\langle\mathrm{x}_{i} \widetilde{\mathrm{x}}_{j}\right\rangle=\left\langle\widetilde{\mathrm{x}}_{j} \mathrm{x}_{i}\right\rangle=G_{j i}, \quad \forall i>j \in \mathbf{Z}
$$

If $K$ is invertible, this output process is identically correlated with the noise, corresponding to the correlation coefficient $r=1$, and in the classical case $\widetilde{K}=K$ such the sequence $\left(\widetilde{\mathrm{x}}_{j}\right)$ is always identified with $\left(\mathrm{x}_{j}\right)$. The invertible case corresponds to the thermal noise which we call standard if $G_{i j}=\delta_{i j}$, that is if the inverse noise $\left(\widetilde{\mathrm{x}}_{j}\right)$ is $\delta$-correlated with $\left(\mathrm{x}_{j}\right)$ such that $\widetilde{K}=K^{-1}$. The opposite situation $\widetilde{K} K=0$ when $\left(\widetilde{\mathrm{x}}_{j}\right)$ is uncorrelated with $\left(\mathrm{x}_{j}\right)$ such that $r=0$ is possible only in the quantum case. It corresponds to the vacuum noise which we call also standard if $K+\widetilde{K}=I$. 
Let us prove the existence of the time-reversed sequence $\widetilde{\mathrm{x}} \neq \mathrm{x}$ in the second order quantum theory corresponding to the case $\widetilde{K} \neq K$, and find the adjoint involution $|\tilde{z}\rangle \mapsto|\tilde{z}\rangle^{b}=\left|\tilde{z}^{\dagger}\right\rangle$,

$$
\left|\sum \zeta_{j} \widetilde{\mathrm{x}}_{j}\right\rangle=\sum \zeta_{j}^{*}\left|\widetilde{\mathrm{x}}_{j}\right\rangle \quad \forall \zeta_{j} \in \mathbf{C}
$$

such that $|\tilde{z}\rangle^{b b}=|\tilde{z}\rangle$, giving the reversed vector representation of the Hermitian conjugation $\tilde{\mathrm{z}} \mapsto \tilde{\mathrm{z}}^{\dagger}=\sum \zeta_{j}^{*} \widetilde{\mathrm{x}}_{j}$ for the complex combinations $\tilde{\mathrm{z}}=\sum \zeta_{j} \widetilde{\mathrm{x}}_{j}$ in the case of invertible $K$.

The commuting matrices $K$ and $\widetilde{K}$ have common eigen vectors $u$. (generalized row-vectors), given as complex eigen-sequences $u=\left(u_{j}\right)_{j \in \mathbf{Z}}$ such that

$$
\sum_{i \in \mathbf{Z}} u_{i} K_{i j}=\kappa u_{j}, \quad \sum_{i \in \mathbf{Z}} u_{i} \widetilde{K}_{i j}=\widetilde{\kappa} u_{j}, \quad j \in \mathbf{Z}
$$

with eigenvalues $\kappa$ and $\widetilde{\kappa} \geq 0$, corresponding to the complex conjugate eigen sequences $\bar{u} .=\left(\bar{u}_{j}\right)_{j \in \mathbf{Z}}$ :

$$
\sum_{i \in \mathbf{Z}} \bar{u}_{i} K_{i j}=\widetilde{\kappa} \bar{u}_{j}, \quad \sum_{i \in \mathbf{Z}} \bar{u}_{i} \widetilde{K}_{i j}=\kappa \bar{u}_{j}, \quad j \in \mathbf{Z}
$$

Indeed, let $K$ and $\widetilde{K}$ have a common eigen-row-vector $u$. with eigenvalues $\kappa \geq 0$ and $\widetilde{\kappa} \geq 0$ respectively. Because $\widetilde{K}$ is the complex conjugate matrix of $K$, $\bar{u}$. is also a common eigenvector, but with the real eigenvalue $\widetilde{\kappa}$ for $K$ and $\kappa$ for $\widetilde{K}$. Two cases arise

$\kappa=\bar{\kappa}:$ In this case we may assume without loss of generality that $u_{j}=$ $\bar{u}_{j}, \forall j \in \mathbf{Z}$

$\kappa \neq \tilde{\kappa}$ : In this case the eigenvectors $u ., \bar{u}$. are orthogonal and so we have $\sum u_{j}^{2}=0$.

This means that the index set $\Omega$ of the eigen sequences $\langle\nu|:=\left(u_{j}(\nu)\right)_{j \in \mathbf{Z}}$ is equipped with a natural flip

$$
\nu \mapsto-\nu, \quad-(-\nu)=\nu, \quad \widetilde{\kappa}(\nu)=\kappa(-\nu)
$$

such that $\bar{u}(\nu)=u$. $(-\nu)$ and the (generalized) Plansherel measure $\mathrm{d} \nu$ on $\Omega$ is invariant under this flip. The complete (with respect to $\mathrm{d} \nu$ ) orthogonal set $\{u .(\nu) \mid \nu \in \Omega\}$ can be chosen in such a way that $u_{j}(\nu)=u_{j}(-\nu)$ iff $\kappa(\nu)=\kappa(-\nu)$ and $\sum_{j \in \mathbf{Z}} u_{j}^{2}(\nu)=0$ iff $\kappa(\nu) \neq \kappa(-\nu)$. This flip defined an isometric involution $\varphi^{\star}(\nu):=\bar{\varphi}(-\nu) \equiv \widetilde{\varphi}^{*}(\nu)$ in the Hilbert space $L^{2}(\Omega)$ as antilinear map $\varphi \mapsto \varphi^{\star}, \varphi^{\star \star}=\varphi$ satisfying

$$
\left\langle\varphi^{\star} \mid \psi\right\rangle=\int \varphi(-\nu) \psi(\nu) \mathrm{d} \nu=\int \varphi(\nu) \psi(-\nu) \mathrm{d} \nu=\left\langle\psi^{\star} \mid \varphi\right\rangle \quad \forall \varphi, \psi \in L^{2}(\Omega) .
$$

We shall assume without loss of generality that the Plansherel measure $\mathrm{d} \nu$ on the null subset $\mathrm{N}_{0}=\{\nu: \kappa(\nu)=0=\widetilde{\kappa}(\nu)\}$ is zero, taking $\mathrm{N}_{0}=\emptyset$ such that its support $\Omega$ is identified with the union $\mathrm{N}_{+}^{\perp} \cup \mathrm{N}_{-}^{\perp}$, where

$$
\mathrm{N}_{+}^{\perp}=\{\nu: \kappa(\nu)>0\}, \mathrm{N}_{-}^{\perp}=\{\nu: \widetilde{\kappa}(\nu)>0\} .
$$

are the complementary subsets of the null sets $\mathrm{N}_{+}$and $\mathrm{N}_{-}$for $\kappa \in L^{1}\left(\mathrm{~N}_{+}^{\perp}\right)$ and $\widetilde{\kappa} \in L^{1}\left(\mathrm{~N}_{-}^{\perp}\right)$. Let us prove that the pairs $\left\{\Omega \ni \nu \mapsto \check{x}_{j}(\nu)\right\} \in L^{2}\left(\mathrm{~N}_{+}^{\perp}\right)$, 
$\left\{\Omega \ni \nu \mapsto \hat{x}_{j}(\nu)\right\} \in L^{2}\left(\mathrm{~N}_{-}^{\perp}\right)$ of complex amplitudes

$$
\check{x}_{j}(\nu)=\kappa(\nu)^{1 / 2} u_{j}(\nu) \equiv\left\langle\nu \mid \mathrm{x}_{j}\right\rangle, \quad \hat{x}_{j}(\nu)=\widetilde{\kappa}(\nu)^{1 / 2} u_{j}(\nu) \equiv\left\langle\nu \mid \widetilde{\mathrm{x}}_{j}\right\rangle
$$

which are related by the isometric involution $\star$ as

$$
\hat{x}_{j}=\kappa(-\nu)^{1 / 2} u_{j}(\nu)=\kappa(-\nu)^{1 / 2} u_{j}^{*}(-\nu)=\check{x}_{j}^{\star}
$$

where $u^{*}=\bar{u}$, give the spectral representation of the quantum noise vectors $\left|\mathrm{x}_{j}\right\rangle$ and $\left|\widetilde{\mathrm{x}}_{j}\right\rangle$.

Indeed, by virtue of the completeness of $\{u .(\nu) \mid \nu \in \Omega\}$ it follows that

$$
\begin{aligned}
& \check{x}_{i}^{\dagger} \check{x}_{j}:=\int \check{x}_{i}(\nu)^{*} \check{x}_{j}(\nu) \mathrm{d} \nu=\int \kappa(\nu) \bar{u}_{i}(\nu) u_{j}(\nu) \mathrm{d} \nu=K_{i j} \\
& \hat{x}_{i}^{\dagger} \hat{x}_{j}:=\int \hat{x}_{i}(\nu)^{*} \hat{x}_{j}(\nu) \mathrm{d} \nu=\int \widetilde{\kappa}(\nu) \bar{u}_{i}(\nu) u_{j}(\nu) \mathrm{d} \nu=\widetilde{K}_{i j} \\
& \check{x}_{i}^{\dagger} \hat{x}_{j}:=\int \check{x}_{i}(\nu)^{*} \hat{x}_{j}(\nu) \mathrm{d} \nu=\int \gamma(\nu) \bar{u}_{i}(\nu) u_{j}(\nu) \mathrm{d} \nu=G_{i j}
\end{aligned}
$$

where we used the invariance of the measure on $\Omega$ with respect to the flip $\nu \mapsto-\nu$.

Let us define the antilinear maps $|\mathrm{z}\rangle \mapsto|\mathrm{z}\rangle^{\sharp},|\tilde{\mathrm{z}}\rangle \mapsto|\tilde{\mathrm{z}}\rangle^{b}$ of vector conjugation

$$
\ddot{z}^{\sharp}=\lambda^{-1 / 2} \check{z}^{\star}, \quad \hat{z}^{b}=\lambda^{1 / 2} \hat{z}^{\star},
$$

respectively on the dense domains $\mathcal{D}_{\sharp} \subset L^{2}\left(\mathrm{~N}_{+}^{\perp}\right)$ and $\mathcal{D}_{\mathrm{b}} \subset L^{2}\left(\mathrm{~N}_{-}^{\perp}\right)$ as the subspaces

$$
\mathcal{D}_{\sharp}=\left\{\check{z}: \lambda^{1 / 2} \check{z} \in L^{2}(\Omega)\right\}, \mathcal{D}_{b}=\left\{\hat{z}: \lambda^{-1 / 2} \hat{z} \in L^{2}(\Omega)\right\}
$$

of square integrable complex amplitudes $\check{z} \in L^{2}(\Omega)$ with the support in $\mathrm{N}_{+}^{\perp}$ and $\hat{z} \in L^{2}(\Omega)$ with the support in $\mathrm{N}_{-}^{\perp}$ where $\lambda^{1 / 2}=\sqrt{\widetilde{\kappa} / \kappa}$ and $\lambda^{-1 / 2}=\sqrt{\kappa / \widetilde{\kappa}}$ are well defined as positive operators of multiplications respectively by $[\widetilde{\kappa}(\nu) / \kappa(\nu)]^{1 / 2}$ and $[\kappa(\nu) / \widetilde{\kappa}(\nu)]^{1 / 2}$ into the subspace $L^{2}(\Theta)$ the common support

$$
\Theta=\{\nu: \kappa(\nu) \neq 0 \neq \widetilde{\kappa}(\nu)\}=\mathrm{N}_{+}^{\perp} \cap \mathrm{N}_{-}^{\perp} .
$$

Note that $\mathcal{D}_{\sharp} \subseteq L^{2}(\Omega)$ is generated by the complex amplitudes $\check{z}=\sum \zeta_{j} \check{x}_{j}$ with $\check{z}^{\star} \in \mathcal{D}_{\mathrm{b}}$ and the adjoint conjugation on the subspace $\mathcal{D}_{\mathrm{b}} \subseteq L^{2}(\Omega)$ is generated by $\hat{z}=\sum \zeta_{j} \hat{x}_{j}$ with $\hat{z}^{\star} \in \mathcal{D}_{\sharp}$ such that $\mathcal{D}_{\sharp}=\mathcal{D}_{b}^{\star}$. Moreover, $\ddot{z}^{\sharp \star}=z^{\star b}, \hat{z}^{b \star}=\hat{z}^{\star \sharp}$ and $\hat{z}^{b}=\lambda \check{z}^{\sharp}, \check{z}^{\sharp}=\lambda^{-1} \check{z}^{b}$ are densely defined involutions in the subspace $L^{2}(\Theta)$ and $\lambda^{\star}=\lambda^{-1}$ on $\Theta$.

In the case of nonzero temperature, when $\Theta=\Omega$ and $\mathcal{D}_{\sharp}, \mathcal{D}_{b}$ are dense in $L^{2}(\Omega)$, the spectral representations of quantum noise and the output process are connected by the spectral linear filters

$$
\hat{x}(\nu)=\lambda(\nu)^{1 / 2} \check{x}(\nu), \quad \check{x}(\nu)=\lambda(\nu)^{-1 / 2} \hat{x}(\nu),
$$

and $\check{x}_{j}^{\sharp}=\check{x}_{j}, \hat{x}_{j}^{b}=\hat{x}_{j}$ for the generating spectral sequences $\left(\check{x}_{j}\right)$ and $\left(\hat{x}_{j}\right)$ :

$$
\begin{array}{cc}
\check{x}_{j}^{\sharp}(\nu)=\lambda(\nu)^{-1 / 2} \hat{x}_{j}(\nu)=\check{x}_{j}(\nu), \quad \nu \in \mathrm{N}_{-}^{\perp}, \\
\hat{x}_{j}^{b}(\nu)=\lambda(\nu)^{1 / 2} \check{x}_{j}(\nu)=\hat{x}_{j}(\nu), \quad \nu \in \mathrm{N}_{+}^{\perp} .
\end{array}
$$


The latter implies $\left|\mathrm{z}^{\dagger}\right\rangle=|\mathrm{z}\rangle^{\sharp},\left|\tilde{\mathrm{z}}^{\dagger}\right\rangle=|\tilde{\mathrm{z}}\rangle^{\mathrm{b}}$ for the complex-linear combinations $\mathrm{z}=$ $\sum \zeta_{i} \mathrm{x}_{i}, \widetilde{\mathrm{z}}=\sum \zeta_{i} \widetilde{\mathrm{x}}_{i}$ since

$$
\begin{aligned}
& \left\langle\mathrm{x}_{i}^{\dagger} \mid \mathrm{x}_{j}^{\dagger}\right\rangle=\left\langle\mathrm{x}_{i} \mid \mathrm{x}_{j}\right\rangle=\left\langle\widetilde{\mathrm{x}}_{j} \mid \widetilde{\mathrm{x}}_{i}\right\rangle=\hat{x}_{j}^{\dagger} \hat{x}_{i}=\check{x}_{i}^{\dagger} \check{x}_{j}=\check{x}_{i}^{\sharp \dagger} \check{x}_{j}^{\sharp}, \\
& \left\langle\widetilde{\mathrm{x}}_{i}^{\dagger} \mid \widetilde{\mathrm{x}}_{j}^{\dagger}\right\rangle=\left\langle\widetilde{\mathrm{x}}_{i} \mid \widetilde{\mathrm{x}}_{j}\right\rangle=\left\langle\mathrm{x}_{j} \mid \mathrm{x}_{i}\right\rangle=\check{x}_{j}^{\dagger} \check{x}_{i}=\hat{x}_{i}^{\dagger} \check{x}_{j}=\hat{x}_{i}^{\dagger \dagger} \hat{x}_{j}^{\dagger}, \\
& \left\langle\widetilde{\mathrm{x}}_{i}^{\dagger} \mid \mathrm{x}_{j}^{\dagger}\right\rangle=\left\langle\widetilde{\mathrm{x}}_{i} \mid \mathrm{x}_{j}\right\rangle=\left\langle\mathrm{x}_{j} \mid \widetilde{\mathrm{x}}_{i}\right\rangle=\check{x}_{j}^{\dagger} \hat{x}_{i}=\hat{x}_{i}^{\dagger} \check{x}_{j}=\hat{x}_{i}^{\dagger \dagger} \check{x}_{j}^{\sharp} .
\end{aligned}
$$

The spectral amplitudes $\check{x}_{j}, \hat{x}_{j}$ in the time representation

$$
x_{j}{ }^{i}=\int \bar{u}_{i}(\nu) \check{x}_{j}(\nu) \mathrm{d} \nu, \quad \widetilde{x}_{j}{ }^{i}=\int \bar{u}_{i}(\nu) \hat{x}_{j}(\nu) \mathrm{d} \nu
$$

describe the canonical vector realization $x_{j}, \widetilde{x}_{j}$ of $\left|\mathrm{x}_{j}\right\rangle,\left|\widetilde{\mathrm{x}}_{j}\right\rangle$. It is given by the matrix elements of the square roots $X=K^{1 / 2}$ and $\widetilde{X}=\widetilde{K}^{1 / 2}$ as

$$
x_{j}{ }^{i}=X_{i j}, \quad \widetilde{x}_{j}{ }^{i}=\widetilde{X}_{i j}, \quad i, j \in \mathbf{Z}
$$

such that $\widetilde{x}_{j}^{i}=\bar{x}_{j}{ }^{i}$ as $\tilde{X}=\bar{X}$. They are thought as the $\ell^{2}(\mathbf{Z})$ columns

$$
x_{j}=\left(x_{j}{ }^{i}\right)^{i \in \mathbf{Z}}, \quad \widetilde{x}_{j}=\left(\bar{x}_{j}{ }^{i}\right)^{i \in \mathbf{Z}}
$$

with the scalar products $x_{i}^{\dagger} x_{j}=\left\langle\mathrm{x}_{i} \mid \mathrm{x}_{j}\right\rangle, \widetilde{x}_{i}^{\dagger} \widetilde{x}_{j}=\left\langle\widetilde{\mathrm{x}}_{i} \mid \widetilde{\mathrm{x}}_{j}\right\rangle$ :

$$
x_{i}^{\dagger} x_{j}=\sum_{k \in \mathbf{Z}} \bar{X}_{k i} X_{k j}=K_{i j}=\sum_{k \in \mathbf{Z}} X_{k i} \bar{X}_{k j}=\widetilde{x}_{j}{ }^{\dagger} \widetilde{x}_{i}, \quad x_{i}{ }^{\dagger} \widetilde{x}_{j}=G_{i j} .
$$

Hence the inversion $x \mapsto \widetilde{x}$ is represented by the usual complex conjugation $\widetilde{x}=$ $\bar{x}, \bar{x}_{j}=\left(\bar{x}_{j}{ }^{i}\right)^{i \in \mathbf{Z}}$, which coincides with transposition : $\bar{x}_{j}{ }^{i}=x_{i}{ }^{j}$ due to the selfadjointness of the square roots of $K=K^{\dagger}$ and so $\bar{K}=\bar{K}^{\dagger}$. Although the vectors $x_{j}, \widetilde{x}_{j}$ seem to be complex if $\widetilde{x}_{j} \neq x_{j}$ due to $x_{j}^{*}:=\bar{x}_{j}=\widetilde{x}_{j}$, they are selfadjoint $x_{j}^{\sharp}=x_{j}, \quad \widetilde{x}_{j}{ }^{\mathrm{b}}=\widetilde{x}_{j}$ in the case $\Theta=\Omega$ with respect to the involution $z^{\sharp}=L^{-1 / 2} z^{*}, \widetilde{z}^{b}=L^{1 / 2} \widetilde{z}^{*}$, where $z^{*}=\bar{z}$ :

$$
L^{1 / 2} x_{j}=\sum L_{k}^{1 / 2} x_{j}{ }^{k}=\widetilde{x}_{j}, \quad L^{-1 / 2} \widetilde{x}_{j}=\sum L^{-1 / 2}{ }_{k} x_{j}{ }^{k}=x_{j}
$$

are the linear input-output and reversed filters in the time representation. The operator $L=\bar{K} K^{-1}$ with the matrix elements

$$
L_{i j}=\int \bar{u}_{i}(\nu) \lambda(\nu) u_{j}(\nu) \mathrm{d} \nu, \quad \lambda(\nu)=\widetilde{\kappa}(\nu) / \kappa(\nu)
$$

is characterized by the properties $L>0, \bar{L}=L^{-1}$ and is called the modular operator in this invertible case. It can be described by the adjoint involutions as $L|\mathrm{z}\rangle=|\mathrm{z}\rangle^{\sharp b}$, and its algebraic analogue is the main object of study in Tomita-Takesaki theory. Note that if $K$ is not invertible, the canonical realizations of $\mathrm{x}$ and $\widetilde{\mathrm{x}}$ are still connected by complex conjugation as the isometric involution $\mathrm{z} \mapsto \mathrm{z}^{*}$, although it may not be described by the linear filtering because the adjoint involutions $\sharp, b$ may not be represented in $\mathcal{E}$. In contrast to the classical theory the constructed canonical realization $\widetilde{x}_{j}$ of the reversed quantum process does not coincide with $x_{j}$ and is even orthogonal to the real quantum noise if $\mathrm{N}_{+} \cup \mathrm{N}_{-}=\Omega$. 
In the case of a stationary time sequence $x_{j}=\sqrt{\varepsilon} x\left(t_{j}\right), t_{j}=\varepsilon j$ the spectrum $\Omega$ is in the interval $\left[-\varepsilon^{-1} / 2, \varepsilon^{-1} / 2\right] \subset \mathbf{R}$, the flip $\nu \mapsto-\nu$ is the usual reflection, and

$$
u_{-j}(\nu)=\varepsilon^{1 / 2} \exp \left\{2 \pi \mathrm{i} \nu t_{j}\right\}=\overline{u_{j}}(\nu) .
$$

The canonical vector realizations of the stationary sequences $\left(x_{j}\right)$ and $\left(\widetilde{x}_{j}\right)$ is given in the time representation by the matrix elements $x_{j}{ }^{i}=\chi_{j-i}, \quad \widetilde{x}_{j}{ }^{i}=\widetilde{\chi}_{j-i}$, where the complex sequences

$$
\begin{aligned}
& \chi_{j}=\varepsilon^{1 / 2} \int_{\Omega} \kappa(\nu)^{1 / 2} \exp \left\{-2 \pi \mathrm{i} \nu t_{j}\right\} \mathrm{d} \nu=\int_{\Omega} x_{j}(\nu) \mathrm{d} \nu \\
& \tilde{\chi}_{j}=\varepsilon^{1 / 2} \int_{\Omega} \widetilde{\kappa}(\nu)^{1 / 2} \exp \left\{-2 \pi \mathrm{i} \nu t_{j}\right\} \mathrm{d} \nu=\int_{\Omega} \widetilde{x}_{j}(\nu) \mathrm{d} \nu
\end{aligned}
$$

are connected by the usual time reflection $\widetilde{x}\left(t_{j}\right)=x\left(-t_{j}\right)$ with respect to $t=0$ : $\tilde{\chi}_{j}=\chi_{-j}$. If $K$ is invertible, this time reflection is described by the stationary input-output and reversed linear complex filters

$$
\chi_{-i}=\sum_{j \in \mathbf{Z}} l^{1 / 2}{ }_{i-j} \chi_{j}, \quad \chi_{i}=\sum_{j \in \mathbf{Z}} l^{-1 / 2}{ }_{i-j} \chi_{-j},
$$

where $l^{1 / 2}{ }_{i-j}=L^{1 / 2}{ }_{i j}, \quad l^{-1 / 2}{ }_{i-j}=L^{-1 / 2}{ }_{i j}$ are given by the integrals

$$
l_{\Theta}{ }_{j}^{1 / 2}:=\varepsilon \int_{\Theta} \lambda(\nu)^{1 / 2} \exp \left\{2 \pi \mathrm{i} \nu t_{j}\right\} \mathrm{d} \nu, \quad l_{\Theta}{ }_{j}^{-1 / 2}:=\varepsilon \int_{\Theta} \lambda(\nu)^{-1 / 2} \exp \left\{2 \pi \mathrm{i} \nu t_{j}\right\} \mathrm{d} \nu
$$

over the support $\Theta \subseteq \Omega$ of $\gamma=(\kappa \widetilde{\kappa})^{1 / 2}$ in the general case.

\section{Spectral Decomposition and Linear Filtering}

Let us consider the stationary quantum noise $\mathrm{x}: t \mapsto \mathrm{x}(t)$ with continuous time $t \in \mathbf{R}$. It can be treated as the limit as $\varepsilon \rightarrow 0$ of a stationary sequence $\varepsilon^{-1 / 2} \mathrm{x}^{\varepsilon}{ }_{j}$ with correlations $k^{\varepsilon}{ }_{j}=\varepsilon k(t)$, given by a complex positive-definite function

$$
k(t)=\int_{-\infty}^{\infty} \exp \{2 \pi \mathrm{i} \nu t\} \kappa(\nu) \mathrm{d} \nu=\langle\mathrm{x}(t) \mid \mathrm{x}(0)\rangle .
$$

This may exist only as a generalized function(distribution) if the support $\mathrm{N}_{+}^{\perp} \subseteq \mathbf{R}$ of the spectral density $\kappa$ is unbounded. The reversed noise $\widetilde{\mathrm{x}}: t \mapsto \widetilde{\mathrm{x}}(t)$, corresponding to the spectral density $\widetilde{\kappa}(\nu)=\kappa(-\nu)$, is described up to second order by the autocorrelation function

$$
\widetilde{k}(t)=\int_{-\infty}^{\infty} \exp \{2 \pi \mathrm{i} \nu t\} \widetilde{\kappa}(\nu) \mathrm{d} \nu=\langle\widetilde{\mathrm{x}}(t) \mid \widetilde{\mathrm{x}}(0)\rangle
$$

and by the symmetric cross-correlation function

$$
r(t)=\int_{-\infty}^{\infty} \exp \{2 \pi \mathrm{i} \nu t\} \gamma(\nu) \mathrm{d} \nu=\langle\mathrm{x}(t) \mid \widetilde{\mathrm{x}}(0)\rangle,
$$

where $\gamma(\nu)=[\widetilde{\kappa}(\nu) \kappa(\nu)]^{1 / 2}$, so that $r$ is the convolutional square root of

$$
[\widetilde{k} * k](t)=\int_{-\infty}^{\infty} k(s-t) k(s) \mathrm{d} s
$$


If $\widetilde{k} * k=0$, i.e. if $\widetilde{\kappa} \kappa=0$, and so $\mathrm{N}_{+}^{\perp} \cap \mathrm{N}_{-}^{\perp}=\emptyset$ we have stationary vacuum noise. The stationary noise is called standard vacuum noise if $k(t)+\widetilde{k}(t)$ is the Dirac $\delta$-function, i.e. if $\widetilde{\kappa}+\kappa=1$.

Such a noise is purely nonclassical because the condition $\widetilde{\kappa} \kappa=0$ for a symmetric function $\widetilde{\kappa}=\kappa$ is only possible in the trivial case $\mathrm{N}_{+}^{\perp}=\emptyset=\mathrm{N}_{-}^{\perp}$ when $\mathrm{x}=0=\widetilde{\mathrm{x}}$.

In the general case the subset $\Theta=\mathrm{N}_{+}^{\perp} \cap \mathrm{N}_{-}^{\perp}$ of $\Omega$ is not empty, but the complement $\Theta^{\perp}=\Omega \backslash \Theta$ can be decomposed into the disjoint union of

$$
\mathrm{N}_{-}=\{\nu \in \Omega \mid \widetilde{\kappa}(\nu)=0\} \subseteq \mathrm{N}_{+}^{\perp}, \quad \mathrm{N}_{+}=\{\nu \in \Omega \mid \kappa(\nu)=0\} \subseteq \mathrm{N}_{-}^{\perp}, \quad \mathrm{N}_{+} \cap \mathrm{N}_{-}=\emptyset .
$$

This means that the quantum noise and its time-reversed version can be uniquely decomposed in the correlation theory into sums $\mathrm{x}_{o}+\mathrm{x}_{\Theta}, \widetilde{\mathrm{x}}_{o}+\widetilde{\mathrm{x}}_{\Theta}$ of uncorrelated vacuum $\left(\mathrm{x}_{o}, \widetilde{\mathrm{x}}_{o}\right)$ and thermal $\left(\mathrm{x}_{\Theta}, \widetilde{\mathrm{x}}_{\Theta}\right)$ components. In the spectral representation they are given by

$$
\begin{gathered}
\check{x}_{o}(t)=P_{-}^{\perp} \check{x}(t), \quad \hat{x}_{o}(t)=P_{+}^{\perp} \hat{x}(t), \\
\check{x}_{\Theta}(t)=P_{\Theta} \check{x}(t), \quad \hat{x}_{\Theta}(t)=P_{\Theta} \hat{x}(t), \quad \forall t \in \mathbf{R}
\end{gathered}
$$

where $P_{+}^{\perp}$ and $P_{-}^{\perp}$ are the projectors on $L^{2}\left(\mathrm{~N}_{+}\right)$and $L^{2}\left(\mathrm{~N}_{-}\right)$and $P_{\Theta}=P_{+} P_{-}$is the orthoprojector on the subspace $L^{2}(\Theta)$. The orthoprojectors $P_{+}=1-1_{+}$and $P_{-}=1-1_{-}$define the best input-output and output-input linear estimates

$$
\begin{gathered}
P_{+} \hat{x}(t)=1_{\Theta} \hat{x}(t)=\lambda_{\Theta}{ }^{1 / 2} \check{x}(t) \text { of } \hat{x}(t)=\lambda_{\Theta}{ }^{1 / 2} \check{x}(t)+\hat{x}_{o}(t), \\
P_{-} \check{x}(t)=1_{\Theta} \check{x}(t)=\lambda_{\Theta}{ }^{-1 / 2} \hat{x}(t) \text { of } \check{x}(t)=\lambda_{\Theta}{ }^{-1 / 2} \hat{x}(t)+\check{x}_{o}(t)
\end{gathered}
$$

where $1_{\Theta}$, defined by $1_{\Theta}(\nu)=1$ if $\nu \in \Theta, 1_{\Theta}(\nu)=0$ if $\nu \notin \Theta$, is the characteristic function of $\Theta=\mathrm{N}_{+}^{\perp} \cap \mathrm{N}_{-}^{\perp}, 1_{+}$and $1_{-}$are the characteristic functions of $\mathrm{N}_{+}$and $\mathrm{N}_{-}$, and

$$
\begin{aligned}
& \lambda_{\Theta}(\nu)^{-1 / 2}=(\sigma(\nu) / \widetilde{\sigma}(\nu)) 1_{\Theta}(\nu) \\
& \lambda_{\Theta}(\nu)^{1 / 2}=(\widetilde{\sigma}(\nu) / \sigma(\nu)) 1_{\Theta}(\nu) .
\end{aligned}
$$

In the time representation

$$
\begin{gathered}
x(t)=\{\chi(t-s) \mid s \in \mathbf{R}\}, \quad \widetilde{x}(t)=\{\widetilde{\chi}(t-s) \mid s \in \mathbf{R}\} \\
\chi(t-s)=\int_{\Omega} \kappa(\nu)^{1 / 2} \exp \{2 \pi \mathrm{i} \nu(s-t)\} \mathrm{d} \nu=\widetilde{\chi}(s-t)
\end{gathered}
$$

$x_{\Theta}(t)$ and $\widetilde{x}_{\Theta}(t)$ are obtained by the replacing the interval $\Omega$ of integration in $\chi=\chi_{\Omega}$ and $\tilde{\chi}=\tilde{\chi}_{\Omega}$ by $\Theta$, with $x_{o}(t)$ given by $\chi_{\mathrm{N}_{-}}$and $\widetilde{x}_{o}(t)$ given by $\chi_{\mathrm{N}_{+}}$. The optimal input-output and output-input filters are given in the Fourier representation by the complex stationary linear nonadapted integrals

$$
\begin{aligned}
& {\left[P_{+} \widetilde{x}(t)\right](s)=\int_{-\infty}^{\infty} l_{\Theta}^{1 / 2}(s-r) \chi(t-r) \mathrm{d} r \equiv\left(l_{\Theta}^{1 / 2} * x\right)(s)} \\
& {\left[P_{-} x(t)\right](s)=\int_{-\infty}^{\infty} l_{\Theta}^{-1 / 2}(s-r) \widetilde{\chi}(t-r) \mathrm{d} r \equiv\left(l_{\Theta}^{-1 / 2} * \widetilde{x}\right)(s) .}
\end{aligned}
$$

Here $l_{\Theta}{ }^{1 / 2}(s-t)=L_{\Theta}{ }^{1 / 2}(s, t), l_{\Theta}^{-1 / 2}(s-t)=L_{\Theta}{ }^{-1 / 2}(s, t)$ are the complex positive definite generalized functions

$$
l_{\Theta}{ }^{1 / 2}(t)=\int \lambda_{\Theta}(\nu)^{1 / 2} \exp \{2 \pi \mathrm{i} \nu t\} \mathrm{d} \nu, \quad l_{\Theta}^{-1 / 2}(t)=\int \lambda_{\Theta}(\nu)^{-1 / 2} \exp \{2 \pi \mathrm{i} \nu t\} \mathrm{d} \nu
$$

characterized by the modular property

$$
l_{\Theta}^{1 / 2}(-t)=\bar{l}_{\Theta}^{1 / 2}(t)=l_{\Theta}^{-1 / 2}(t) .
$$


The case $\mathrm{N}_{+}=\emptyset=\mathrm{N}_{-}$corresponds to the purely thermal noise which is called standard thermal noise, if $\widetilde{\kappa} \kappa=1$ in a given spectral region $\Omega \subseteq \mathbf{R}$. If $\Omega=\mathbf{R}$, it can be written as $[\widetilde{k} * k](t)=\delta(t)$ in terms of the Dirac correlation function $r(t)=\delta(t)$.

The thermal noise is called white if the spectrum $\kappa$ and thus also $\widetilde{\kappa}$ is flat: $\kappa(\nu)=\sigma^{2}=\widetilde{\kappa}(\nu), \forall \nu \in \Omega$. White noise coincides with its time-reversed version and is essentially classical (at least in second order). There exists just one such standard noise $\mathrm{x}=\mathrm{w}=\widetilde{\mathrm{x}}$ described in the second order theory by the correlation function $k(t)=\delta(t)=\bar{k}(t)$.

In the quantum case there are many standard thermal noises and $\delta$-correlated with them reversed version $\tilde{\chi}(t)=\chi(-t)$. They are parametrized by a modular spectral function

$$
\lambda(\nu)>0, \widetilde{\lambda}(\nu)=\lambda(-\nu)=\lambda(\nu)^{-1}, \forall \nu \in \Omega
$$

giving the standard correlation functions $k, \bar{k}$ as the convolution square roots

$$
k(t)=l^{-1 / 2}(t), \bar{k}(t)=l^{1 / 2}(t), \forall t \in \mathbf{R}
$$

Any stationary quantum process $\mathrm{y}: t \rightarrow \mathrm{y}(t)$ and its reverse $\widetilde{\mathrm{y}}: t \rightarrow \widetilde{\mathrm{y}}(t)$ described up to second order by the correlation function

$$
\langle\mathrm{y}(t) \mathrm{y}(0)\rangle=\int \exp \{2 \pi \mathrm{i} \nu t\} \sigma(\nu)^{2} \mathrm{~d} \nu=\langle\widetilde{\mathrm{y}}(0) \widetilde{\mathrm{y}}(t)\rangle
$$

can be obtained by stationary filtering of standard quantum noises $\mathrm{x}$ and $\widetilde{\mathrm{x}}$, i.e. such that their spectral densities

$$
\kappa(\nu)=1_{-}(\nu)+\lambda_{\Theta}(\nu)^{-1 / 2}, \widetilde{\kappa}(\nu)=1_{+}(\nu)+\lambda_{\Theta}(\nu)^{1 / 2}
$$

and $\gamma(\nu)=1_{\Theta}(\nu)$. In the spectral representation

$$
\check{y}(t)=\{\check{y}(t, \nu) \mid \nu \in \Omega\}, \quad \hat{y}(t)=\{\hat{y}(t, \nu) \mid \nu \in \Omega\}
$$

the filtering is given by

$$
\check{y}(t, \nu)=f(\nu) \check{x}(t, \nu), \hat{y}(t, \nu)=f(\nu) \hat{x}(t, \nu)
$$

where $\bar{f}=f=\tilde{f}$ is a real symmetric transmission function $f: \Omega \rightarrow \mathbf{R}_{+}$of the standard complex amplitudes

$$
\begin{gathered}
\check{x}(t, \nu)=\exp \{2 \pi \mathrm{i} \nu t\} \check{x}(\nu), \hat{x}(t, \nu)=\exp \{2 \pi \mathrm{i} \nu t\} \hat{x}(\nu) \\
\check{x}(\nu)=1_{-}(\nu)+\lambda_{\Theta}(\nu)^{-1 / 4}, \hat{x}(\nu)=1_{+}(\nu)+\lambda_{\Theta}(\nu)^{1 / 4} .
\end{gathered}
$$

The transmission function $f$ is uniquely defined in the space $L^{2}(\Omega)$ by

$$
f(\nu)=[\sigma(\nu) \widetilde{\sigma}(\nu)]^{1 / 2} 1_{\Theta}(\nu)+[\sigma(\nu) \vee \widetilde{\sigma}(\nu)]^{1 / 2} 1_{\Theta}^{\perp}(\nu) .
$$

In the time representation $y(t)=\{\psi(t-s)\}, \widetilde{y}(t)=\{\widetilde{\psi}(t-s)\}$, given by the Fourier integral

$$
\psi(t)=\int \hat{y}(\nu) \exp \{2 \pi \mathrm{i} \nu t\} \mathrm{d} \nu, \widetilde{\psi}(t)=\int \check{y}(\nu) \exp \{2 \pi \mathrm{i} \nu t\} \mathrm{d} \nu,
$$

the filters are written as real nonadapted stationary transformations

$$
\psi(t-s)=\int_{-\infty}^{\infty} \phi(s-r) \chi(t-r) \mathrm{d} r=\widetilde{\psi}(s-t)
$$


where

$$
\phi(t)=\int f(\nu) \exp \{2 \pi \mathrm{i} \nu t\} \mathrm{d} \nu .
$$

An important example of the stationary quantum thermal noise is given by the Planck's spectral density

$$
\kappa(\nu)=h \nu\left(\mathrm{e}^{\beta h \nu}-1\right)^{-1}, \widetilde{\kappa}(\nu)=h \nu\left(1-\mathrm{e}^{-\beta h \nu}\right)^{-1} .
$$

In this case $\widetilde{\kappa}(\nu)-\kappa(\nu)=h \nu>0$ if $\nu>0$, i.e. $\Theta_{+}=\mathbf{R}_{+}, \lambda(\nu)=\exp \{\beta h \nu\} \neq$ $0 \forall \nu \in \mathbf{R}$, and $\lambda(\nu) \neq 1$ for $\nu \neq 0$, and $\lambda(\nu)=1$ as for the classical white noise only if $\beta=0$. It describes stationary Bose noise in which the electromagnetic (optical) field is in an equilibrium state of the temperature $\theta=1 / \beta$. The modular function for such noise,

$$
l(t)=\int \exp \{2 \pi \mathrm{i} \nu t\} \exp \{\beta h \nu\} \mathrm{d} \nu=\delta\left(t+\frac{\hbar}{\mathrm{i}} \beta\right)=\exp \left\{\frac{\hbar}{\mathrm{i}} \beta \frac{\mathrm{d}}{\mathrm{d} t}\right\} \delta(t),
$$

is extremely singular, as is the correlation function

$$
k(t)=\frac{\hbar}{\mathrm{i}}\left(\exp \left\{\frac{\hbar}{\mathrm{i}} \beta \frac{\mathrm{d}}{\mathrm{d} t}\right\}-1\right)^{-1} \frac{\mathrm{d}}{\mathrm{d} t} \delta(t)
$$

$(\hbar=h / 2 \pi)$ with complex conjugate

$$
\bar{k}(t)=\frac{\hbar}{\mathrm{i}}\left(1-\exp \left\{-\frac{\hbar}{\mathrm{i}} \beta \frac{\mathrm{d}}{\mathrm{d} t}\right\}\right)^{-1} \frac{\mathrm{d}}{\mathrm{d} t} \delta(t)
$$

for the time-reversed Planck noise corresponding to the density $\widetilde{\kappa}$.

Although the cross correlation function

$$
r(t)=\frac{\hbar}{2}\left[\sin \left(\frac{\hbar}{2} \beta \frac{\mathrm{d}}{\mathrm{d} t}\right)\right]^{-1} \frac{\mathrm{d}}{\mathrm{d} t} \delta(t)
$$

is less singular, due to the integrability of its spectral density

$$
\gamma(\nu)=h \nu[\exp \{\beta h \nu / 2\}-\exp \{-\beta h \nu / 2\}]^{-1},
$$

quantum stochastic integrals corresponding to linear stationary filters for such noise are not defined in the usual sense. Indeed, the standard quantum pair $(\mathrm{x}, \widetilde{\mathrm{x}})$ corresponding to the Planck density have the singular correlation functions

$$
l^{-1 / 2}(t)=\delta(t+\mathrm{i} \hbar \beta / 2), \quad l^{1 / 2}(t)=\delta(t-\mathrm{i} \hbar \beta / 2)
$$

and the cross-correlation function $r(t)=\delta(t)$. To obtain the Planck noise y and its time-reversed version $\widetilde{\mathrm{y}}$ one should integrate the standard quantum noise $\mathrm{x}$ and $\widetilde{\mathrm{x}}$ with the non singular filtering function

$$
f(t)=\int[h \nu / 2 \operatorname{sh}(\beta h \nu / 2)]^{1 / 2} \exp \{2 \pi \mathrm{i} \nu t\} \mathrm{d} \nu
$$

But the result of the integration remains singular, corresponding to the singularity of $k$ and $\bar{k}$. 


\section{Quantum Stochastic Integration}

In quantum theory the noise $\mathrm{x}(t)$ is thought of as represented by Hermitian, or even selfadjoint operators in a Hilbert space of state vectors (probability amplitudes), with zero mean values $\phi[\mathrm{x}(t)]=\langle\phi|\mathrm{x}(t)| \phi\rangle=0$. Here $|\phi\rangle$ is a fixed unit vector of the given state $\phi$ which can be always be chosen as vector state represented by the vacuum vector $|\phi\rangle=\delta_{\emptyset}$ in a Fock space $\mathcal{H}=\mathcal{F}$. The quantum correlations $K(s, t)=k(s-t)$ are defined for a stationary state $\phi$ by the scalar product in $\mathcal{H}$,

$$
k(t)=\langle\phi|\mathrm{x}(t) \mathrm{x}(0)| \phi\rangle \equiv \phi[\mathrm{x}(t) \mathrm{x}(0)] .
$$

If $k(t)$ is complex, the operator $\mathrm{x}(t)=\mathrm{x}(t)^{\dagger}$ will not commute with $\mathrm{x}(0)=\mathrm{x}(0)^{\dagger}$. The time-reversed correlations $\widetilde{k}(t)=k(-t)$ are "observable" in the real arrow of time only for the time-reversed noise $\widetilde{\mathrm{x}}$ which gives a natural representation in the same Hilbert space $\mathcal{H}$ an output process $\widetilde{\mathrm{x}}: t \mapsto \widetilde{\mathrm{x}}(t)$, for which $\widetilde{k}(t)=\langle\phi|\widetilde{\mathrm{x}}(t) \widetilde{\mathrm{x}}(0)| \phi\rangle$ with the same $\phi \in \mathcal{H}$.

The vector processes $t \mapsto|\mathrm{x}(t)\rangle, t \mapsto|\widetilde{\mathrm{x}}(t)\rangle$ are obtained from the operator representations in $\mathcal{H}$ by the identifications

$$
|\mathrm{x}(t)\rangle:=\mathrm{x}(t)|\phi\rangle \equiv x(t), \quad|\widetilde{\mathrm{x}}(t)\rangle:=\widetilde{\mathrm{x}}(t)|\phi\rangle \equiv \widetilde{x}(t)
$$

in the minimal subspace generated by the set $\{\mathrm{x}(t)|\phi\rangle, \widetilde{\mathrm{x}}(t)|\phi\rangle\} \subset \mathcal{H}$. In a classical theory this gives a one-to-one correspondence between vectors and operators of multiplication in the total space $\mathcal{H}$. This is not the case in quantum probability theory as one can observe for vacuum correlations, for which it is possible that $|\mathrm{z}\rangle=\mathrm{z}|\phi\rangle=0$ for annihilation operators $\mathrm{z} \neq 0$. This is why the vector integration theory is not sufficient in the quantum case; we need an operator form of quantum stochastic integration.

According to the nondemolition (causality) principle of quantum theory [12] the input observables $\mathrm{x}(0)$ must commute with the fundamental output observables $\widetilde{\mathrm{x}}(t)$ for all $t \leq 0$ and they must commute also for $t \geq 0$ because the noise $\mathrm{x}$ can be regarded as the output process for the time-reversed noise $\widetilde{\mathrm{x}}$. At the correlation level this is expressed in terms of the reflection symmetry

$$
\langle\phi|\mathrm{x}(0) \widetilde{\mathrm{x}}(t)| \phi\rangle=r(t)=\langle\phi|\widetilde{\mathrm{x}}(t) \mathrm{x}(0)| \phi\rangle, \quad \forall t \in \mathbf{R}
$$

for the cross correlation function $r(t)=(\widetilde{k} * k)^{1 / 2}(t)$.

Now we are going to develop a second order theory of quantum stochastic integration with respect to any colored quantum noise and its fundamental output process. The realization of such theory was given in 10 in terms of a representation of the canonical commutation relations in the symmetric Fock space $\mathcal{H}$. This provides a one-to-one correspondence between second order integration theory and the Gaussian integration theory of quantum thermal noise with respect to the vacuum state $\phi$. Without loss of generality we may assume that the pare $\mathrm{x}(t), \widetilde{\mathrm{x}}(t)$ is standard, i.e. $\kappa \widetilde{\kappa}=1$.

Let us denote by $\zeta$ the complex test functions $t \mapsto \zeta(t)$, for which the "integrals"

$$
\zeta \cdot \mathrm{x}=\int_{-\infty}^{\infty} \zeta(t) \mathrm{x}(t) \mathrm{d} t, \quad \zeta \cdot \widetilde{\mathrm{x}}=\int_{-\infty}^{\infty} \zeta(t) \widetilde{\mathrm{x}}(t) \mathrm{d} t
$$


are expected to be well defined as selfadjoint operators in $\mathcal{H}$ if $\bar{\zeta}=\zeta$. If $\zeta$ is given as a Fourier integral

$$
\check{c}(t):=\int c(\nu) \exp \{2 \pi \mathrm{i} \nu t\} \mathrm{d} \nu \equiv \hat{c}(-t),
$$

then the operator-valued functionals $\zeta \mapsto \zeta \cdot \mathrm{x}, \zeta \mapsto \zeta \cdot \widetilde{\mathrm{x}}$ can be treated as quantum stochastic integrals

$$
\check{c} \cdot \mathrm{x}=\int c(\nu) \check{\mathrm{X}}(\mathrm{d} \nu), \quad \int c(\nu) \hat{\mathrm{X}}(\mathrm{d} \nu)=\hat{c} \cdot \mathrm{x}
$$

for the Fourier transforms $c=\widehat{\zeta}$ of $\zeta=\check{c}$ and $c=\check{\zeta}$ of $\zeta=\hat{c}$.

The quantum stochastic integrators $\check{X}, \hat{\mathrm{X}}$, defined in 14 as operator-valued measures on the spectral intervals $\Delta \subset \Omega$, in second order are described by spectral vector-measures

$$
|\check{\mathrm{X}}(\Delta)\rangle:=\check{\mathrm{X}}(\Delta)|\phi\rangle \equiv \check{X}(\Delta), \quad|\hat{\mathrm{X}}(\Delta)\rangle:=\hat{\mathrm{X}}(\Delta)|\phi\rangle \equiv \hat{X}(\Delta)
$$

which are related by isometric involution $\star=-*$ on the vector space as $\hat{X}^{\star}=\check{X}$, i.e. $\hat{X}^{*}(-\Delta)=\check{X}(\Delta)$.

They, together with $\check{X}(\Delta)^{\dagger}=\langle\check{\mathrm{X}}(\Delta)|, \hat{X}(\Delta)^{\dagger}=\langle\hat{\mathrm{X}}(\Delta)|$ are assumed to satisfy the following properties:

(i) orthogonal $\sigma$-additivity for disjoint unions $\sum \Delta_{i}=\Delta$

$$
\check{X}(\Delta)=\oplus \check{X}\left(\Delta_{i}\right), \quad \hat{X}(\Delta)=\oplus \hat{X}\left(\Delta_{i}\right) .
$$

(ii) absolute continuity:

$$
\begin{aligned}
\check{X}(\Delta)^{\dagger} \check{X}\left(\Delta^{\prime}\right) & =\int_{\Delta \cap \Delta^{\prime}} \kappa(\nu) \mathrm{d} \nu \\
\hat{X}(\Delta)^{\dagger} \hat{X}\left(\Delta^{\prime}\right) & =\int_{\Delta \cap \Delta^{\prime}} \widetilde{\kappa}(\nu) \mathrm{d} \nu \\
\check{X}(\Delta)^{\dagger} \hat{X}\left(\Delta^{\prime}\right) & =\int_{\Delta \cap \Delta^{\prime}} \gamma(\nu) \mathrm{d} \nu
\end{aligned}
$$

(iii) selfadjointness on $\Theta=\mathrm{N}_{+}^{\perp} \cap \mathrm{N}_{-}^{\perp}$ :

$$
\check{X}(\Delta)^{\sharp}=\lambda^{-1} \cdot \hat{X}(\Delta)=\check{X}(\Delta), \quad \hat{X}(\Delta)^{b}=\lambda \cdot \hat{X}(\Delta)=\hat{X}(\Delta) \quad \Delta \subseteq \Theta,
$$

where $\gamma=(\widetilde{\kappa} \kappa)^{1 / 2}, \lambda(\nu)=(\tilde{\kappa} / \kappa)^{1 / 2}$ and $\lambda \cdot \hat{X}(\Delta)=\int_{\Delta}^{\oplus} \lambda(\nu) \hat{X}(\mathrm{~d} \nu)$. The $\sigma$ additivity makes it possible to define this integral as integral as an orthogonal vector measure $\check{X}$ absolutely continuous with $\hat{X}$ on $\Theta$. The condition (ii) can be symbolically written in the form of a multiplication table

$$
\begin{array}{ll}
\check{X}(\mathrm{~d} \nu)^{\dagger} \check{X}\left(\mathrm{~d} \nu^{\prime}\right)=\delta_{\nu \nu^{\prime}} \kappa(\nu) \mathrm{d} \nu, & \check{X}(\mathrm{~d} \nu)^{\dagger} \hat{X}\left(\mathrm{~d} \nu^{\prime}\right)^{\dagger}=\delta_{\nu \nu^{\prime}} \gamma(\nu) \mathrm{d} \nu \\
\hat{X}(\mathrm{~d} \nu)^{\dagger} \check{X}\left(\mathrm{~d} \nu^{\prime}\right)=\delta_{\nu \nu^{\prime}} \gamma(\nu) \mathrm{d} \nu, & \hat{X}(\mathrm{~d} \nu)^{\dagger} \hat{X}\left(\mathrm{~d} \nu^{\prime}\right)=\delta_{\nu \nu^{\prime}} \widetilde{\kappa}(\nu) \mathrm{d} \nu
\end{array}
$$

where $\delta_{\nu \nu^{\prime}}=0$ if $\nu \neq \nu^{\prime}, \delta_{\nu \nu^{\prime}}=1$ if $\nu=\nu^{\prime}$, and $\hat{X}(\mathrm{~d} \nu)=\lambda(\nu) \check{X}(\mathrm{~d} \nu)$. An operator realization of this table can be given in the Fock space $\mathcal{F}$ with respect to the vacuum state vector $|\phi\rangle=\mathcal{H}[10]$.

Using this multiplication table we obtain the isometry property

$$
\left\langle\phi\left|\mathrm{y}^{\dagger} \mathrm{y}\right| \phi\right\rangle=\int\left|a(\nu) \kappa(\nu)^{1 / 2}+c(\nu) \widetilde{\kappa}(\nu)^{1 / 2}\right|^{2} \mathrm{~d} \nu=b^{\dagger} b
$$


of the map $\mathrm{y} \mapsto b=a \kappa^{1 / 2}+c \widetilde{\kappa}^{1 / 2}$ for the operators $\mathrm{y}=\check{a} \cdot \mathrm{x}+\check{c} \cdot \widetilde{\mathrm{x}}=\eta \cdot \mathrm{x}$ with $\eta=\check{a}+\hat{c}$ on the state vector $\phi$ into the spectral representation $b(\nu)=\langle\nu \mid \mathrm{y}\rangle$ of $|\mathrm{y}\rangle=|\eta \cdot \mathrm{x}\rangle$. This extends the quantum stochastic integral $\eta \cdot \mathrm{x}$ with $b^{\dagger} b=\int|b(\nu)|^{2} \mathrm{~d} \nu<\infty$ from the functions $\eta(t)$ with simple $(a, c)$ to any functions $\eta=\check{a}+\hat{c}$ for which the complex amplitude $b$ remains square integrable. To ensure also $\left\langle\phi\left|\mathrm{yy}^{\dagger}\right| \phi\right\rangle\langle\infty$, we must add the condition of square integrability $b^{\star} \in L^{2}(\Omega)$ for the amplitude $b^{\star}=a^{\star} \kappa^{1 / 2}+c^{\star} \widetilde{\kappa}^{1 / 2}$, that is, we define the quantum stochastic integral y together with $\mathrm{y}^{\dagger}$ only for the test functions $\check{a}, \hat{c} \subset \mathcal{E}$. In the classical case, $\widetilde{\kappa}=\kappa$ when $b^{\star}=b^{*}$, the square integrability of $b$ and $b^{*}$ are equivalent to that of $a$ and $c$ with respect to the symmetric measure $\widetilde{\kappa} \mathrm{d} \nu=\kappa \mathrm{d} \nu$. In the purely quantum case of vacuum noise it is not so, and the conditions $b, b^{\star} \in L^{2}(\Omega)$ are equivalent to the square integrability of $a$ and $c$ with respect to $\kappa \vee \widetilde{\kappa} \mathrm{d} \nu$.

In spectral representation the standard vacuum noise $\mathrm{x}(t)$ together with the reversed process $\widetilde{\mathrm{x}}(t)$ is described by the canonical operator-valued measures

$$
\mathrm{A}^{+}(\Delta)=\left\{\begin{array}{l}
\hat{\mathrm{X}}(\Delta), \Delta \subset \mathrm{N}_{+}^{\perp} \\
\check{\mathrm{X}}(\Delta), \Delta \subset \mathrm{N}_{-}^{\perp}
\end{array} \quad, \quad \mathrm{A}_{-}(\Delta)=\left\{\begin{array}{l}
\check{\mathrm{X}}(\Delta), \Delta \subset \mathrm{N}_{+}^{\perp} \\
\hat{\mathrm{X}}(\Delta), \Delta \subset \mathrm{N}_{-}^{\perp}
\end{array}\right.\right.
$$

of independent creation $\mathrm{A}_{\Delta}^{+}=\mathrm{A}^{+}(\Delta)$ and annihilation $\mathrm{A}_{-}^{\Delta}=\mathrm{A}_{-}(\Delta)$ such that

$$
\left\langle\phi\left|\mathrm{A}_{\Delta}^{+}=0, \mathrm{~A}_{-}^{\Delta}\right| \phi\right\rangle=0 .
$$

It defines the standard operator vacuum measures $\hat{\mathrm{X}}$ and $\check{\mathrm{X}}$ as

$$
\hat{\mathrm{X}}(\Delta)=\mathrm{A}_{-}\left(\Delta \cap \mathrm{N}_{-}^{\perp}\right)+\mathrm{A}^{+}\left(\Delta \cap \mathrm{N}_{+}^{\perp}\right), \quad \check{\mathrm{X}}(\Delta)=\mathrm{A}_{-}\left(\Delta \cap \mathrm{N}_{+}^{\perp}\right)+\mathrm{A}^{+}\left(\Delta \cap \mathrm{N}_{-}^{\perp}\right)
$$

on the intervals $\Delta \subset \Omega$ for the given state $|\phi\rangle \in \mathcal{H}$. The canonical pair $\left(\mathrm{A}_{-}^{0}, \mathrm{~A}_{0}^{+}\right)$of the creation and annihilation measures is characterized by the following properties of flip-adjointness

$$
\mathrm{A}_{-}^{\Delta^{\dagger}}=\mathrm{A}_{-\Delta}^{+} \equiv \mathrm{A}_{+}^{\Delta}, \mathrm{A}_{\Delta}^{+\dagger}=\mathrm{A}_{-}^{-\Delta} \equiv \mathrm{A}_{\Delta}^{-},
$$

and orthogonality of all products apart of $\mathrm{A}_{-}(\Delta) \mathrm{A}_{+}\left(\Delta^{\prime}\right)$ for $\Delta \cap \Delta^{\prime} \neq \emptyset$ :

$$
\begin{gathered}
\left\langle\phi\left|\mathrm{A}_{-}(-\Delta) \mathrm{A}^{+}\left(\Delta^{\prime}\right)\right| \phi\right\rangle=\nu\left(\Delta \cap \Delta^{\prime}\right), \quad\left\langle\phi\left|\mathrm{A}^{+}(-\Delta) \mathrm{A}_{-}\left(\Delta^{\prime}\right)\right| \phi\right\rangle=0, \\
\left\langle\phi\left|\mathrm{A}^{+}(-\Delta) \mathrm{A}^{+}\left(\Delta^{\prime}\right)\right| \phi\right\rangle=0, \quad\left\langle\phi\left|\mathrm{A}_{-}(-\Delta) \mathrm{A}_{-}\left(\Delta^{\prime}\right)\right| \phi\right\rangle=0 .
\end{gathered}
$$

This table can be written also symbolically in the canonical form [6] as

$$
\begin{gathered}
\mathrm{A}_{-}(\mathrm{d} \nu) \mathrm{A}_{+}\left(\mathrm{d} \nu^{\prime}\right)=\delta_{\nu \nu^{\prime}} d \nu, \quad \mathrm{A}^{+}(\mathrm{d} \nu) \mathrm{A}^{-}\left(\mathrm{d} \nu^{\prime}\right)=0, \\
\mathrm{~A}^{+}(\mathrm{d} \nu) \mathrm{A}_{+}\left(\mathrm{d} \nu^{\prime}\right)=0, \quad \mathrm{~A}_{-}(\mathrm{d} \nu) \mathrm{A}^{-}\left(\mathrm{d} \nu^{\prime}\right)=0,
\end{gathered}
$$

for all $\nu, \nu^{\prime} \in \Omega$. This table includes also $\mathrm{A}^{-}(\mathrm{d} \nu) \mathrm{A}^{+}\left(\mathrm{d} \nu^{\prime}\right)=\delta_{\nu \nu^{\prime}} d \nu$ and all other products equal zero by the reflection $\nu \mapsto-\nu$.

Let us prove that an arbitrary (not necessary vacuum) stationary (in the second order sense) quantum process y $(t)$ together with its time-reversed version $\widetilde{y}(t)$ can be obtained by quantum stochastic integration with respect to the flip-selfadjoint canonical pair $\left(\mathrm{A}^{+}, \mathrm{A}_{-}\right)$over $\Omega \subseteq \mathbf{R}$, or, equivalently, with respect to the selfadjoint quadruple $\left(\mathrm{A}^{+}, \mathrm{A}^{-} ; \mathrm{A}_{-}, \mathrm{A}_{+}\right)$on the positive part $\Omega_{+} \subseteq \mathbf{R}_{+}$of $\Omega$.

Indeed, in general the pair

$$
\mathrm{y}(t)=\int \exp \{-2 \pi \mathrm{i} \nu t\} \check{\mathrm{Y}}(\mathrm{d} \nu), \quad \widetilde{\mathrm{y}}(t)=\int \exp \{-2 \pi \mathrm{i} \nu t\} \hat{\mathrm{Y}}(\mathrm{d} \nu)
$$


of operator-valued distributions $\check{c} \mapsto \check{c} \cdot \mathrm{y}, \check{c} \cdot \widetilde{\mathrm{y}}$ is given by a pair $(\check{\mathrm{Y}}, \hat{\mathrm{Y}})$ of operatorvalued orthogonal flip-selfadjoint measures $\hat{\mathrm{Y}}(\mathrm{d} \nu)^{\dagger}=\check{\mathrm{Y}}(-\mathrm{d} \nu)$ with multiplication table

$$
\begin{aligned}
& \check{\mathrm{Y}}(\mathrm{d} \nu) \check{\mathrm{Y}}(\mathrm{d} \nu)^{\dagger}=\sigma(\nu)^{2} \mathrm{~d} \nu, \quad \hat{\mathrm{Y}}(\mathrm{d} \nu) \check{\mathrm{Y}}(\mathrm{d} \nu)^{\dagger}=\widetilde{\sigma}(\nu) \sigma(\nu) \mathrm{d} \nu, \\
& \check{\mathrm{Y}}(\mathrm{d} \nu) \hat{\mathrm{Y}}(\mathrm{d} \nu)^{\dagger}=\sigma(\nu) \widetilde{\sigma}(\nu) \mathrm{d} \nu, \quad \hat{\mathrm{Y}}(\mathrm{d} \nu) \hat{\mathrm{Y}}(\mathrm{d} \nu)^{\dagger}=\widetilde{\sigma}(\nu)^{2} \mathrm{~d} \nu .
\end{aligned}
$$

Such measures can be obtained as quantum stochastic integrals

$$
\begin{aligned}
& \check{\mathrm{Y}}(\Delta)=\int_{\Delta} \sigma(\nu) \mathrm{A}_{-}(\mathrm{d} \nu)+\tilde{\sigma}(\nu) \mathrm{A}^{+}(\mathrm{d} \nu) \\
& \hat{\mathrm{Y}}(\Delta)=\int_{\Delta} \tilde{\sigma}(\nu) \mathrm{A}_{-}(\mathrm{d} \nu)+\sigma(\nu) \mathrm{A}^{+}(\mathrm{d} \nu)
\end{aligned}
$$

from the canonical pair $\left(\mathrm{A}^{+}, \mathrm{A}_{-}\right)$of flip-adjoint annihilation and creation measures. Moreover, in the nonclassical case $\sigma(\nu) \neq \widetilde{\sigma}(\nu), \forall \nu \in \Omega$, corresponding to quantum noise of zero or finite temperature, the canonical pair $\mathrm{A}=\left(\mathrm{A}^{+}, \mathrm{A}_{-}\right)$is uniquely defined over $\Omega$ by the pair $(\check{Y}, \hat{Y})$ from

$$
\begin{aligned}
& \left(\widetilde{\sigma}(\nu)^{2}-\sigma(\nu)^{2}\right) \mathrm{A}^{+}(d \nu)=\widetilde{\sigma}(\nu) \check{\mathrm{Y}}(\mathrm{d} \nu)-\sigma(\nu) \hat{\mathrm{Y}}(\mathrm{d} \nu), \\
& \left(\widetilde{\sigma}(\nu)^{2}-\sigma(\nu)^{2}\right) \mathrm{A}_{-}(\mathrm{d} \nu)=\widetilde{\sigma}(\nu) \hat{\mathrm{Y}}(\mathrm{d} \nu)-\sigma(\nu) \check{\mathrm{Y}}(\mathrm{d} \nu) .
\end{aligned}
$$

This means that the direct and reversed quantum stochastic integrals $\zeta \cdot \mathrm{y}, \zeta \cdot \widetilde{\mathrm{y}}$ can be written for the Fourier integrals of $\zeta=\check{c}$ as

$$
\zeta \cdot \mathrm{y}=\int g(\nu) \mathrm{A}_{-}(\mathrm{d} \nu)+\tilde{g}(\nu) \mathrm{A}^{+}(\mathrm{d} \nu)=\mathrm{A}(\sigma c, \widetilde{\sigma} c)
$$

with $g(\nu)=\sigma(\nu) c(\nu)$ and

$$
\zeta \cdot \widetilde{\mathrm{y}}=\int \tilde{g}(\nu) \mathrm{A}_{-}(\mathrm{d} \nu)+g(\nu) \mathrm{A}^{+}(\mathrm{d} \nu)=\mathrm{A}(\tilde{\sigma} c, \sigma c)
$$

with $\tilde{g}(\nu)=\tilde{\sigma}(\nu) c(\nu)$.

To obtain these integrals in the time representation as linear stationary filters of the adjoint pair $\hat{\mathrm{a}}=\left(\hat{\mathrm{a}}^{+}, \hat{\mathrm{a}}_{-}\right)$of the canonical annihilation $\hat{\mathrm{a}}_{-}(t)$ and creation $\hat{a}^{+}(t)$ distributions

$$
\begin{aligned}
& \hat{\mathrm{a}}_{-}(t)=\int \exp \{-2 \pi \mathrm{i} \nu t\} \mathrm{A}_{-}(\mathrm{d} \nu)=\hat{\mathrm{a}}^{+}(t)^{\dagger}, \\
& \hat{\mathrm{a}}^{+}(t)=\int \exp \{-2 \pi \mathrm{i} \nu t\} \mathrm{A}^{+}(\mathrm{d} \nu)=\hat{\mathrm{a}}_{-}(t)^{\dagger},
\end{aligned}
$$

or their time reversals $\check{\mathrm{a}}^{-}(t)=\hat{\mathrm{a}}_{-}(-t), \check{\mathrm{a}}_{+}(t)=\hat{\mathrm{a}}^{+}(-t)$ we can use the FourierParseval identity

$$
\mathrm{A}\left(\widehat{\varphi}^{-}, \widehat{\varphi}_{+}\right)=\hat{\mathrm{a}}\left(\varphi^{-}, \varphi_{+}\right) .
$$

The latter is given by the standard creation and annihilation integrators

$$
\hat{\mathrm{A}}^{+}(\mathrm{d} t)=\hat{\mathrm{a}}^{+}(t) \mathrm{d} t=\hat{\mathrm{A}}_{-}(\mathrm{d} t)^{\dagger}, \quad \hat{\mathrm{A}}_{-}(\mathrm{d} t)=\hat{\mathrm{a}}_{-}(t) \mathrm{d} t=\hat{\mathrm{A}}^{+}(\mathrm{d} t)^{\dagger}
$$

in the time representation as the standard quantum stochastic integral

$$
\hat{\mathrm{a}}\left(\varphi^{-}, \varphi_{+}\right)=\int_{-\infty}^{\infty} \varphi_{+}(t) \hat{\mathrm{A}}^{+}(\mathrm{d} t)+\varphi^{-}(t) \hat{\mathrm{A}}_{-}(\mathrm{d} t)
$$


Applying this to the quantum stochastic integral in the spectral domain with the general integrands

$$
\begin{aligned}
& f^{-}(\nu)=a(\nu) \widetilde{\sigma}(\nu)+c(\nu) \sigma(\nu)=\widehat{\varphi}^{-}(\nu) \\
& f_{+}(\nu)=a(\nu) \sigma(\nu)+c(\nu) \widetilde{\sigma}(\nu)=\widehat{\varphi}_{+}(\nu)
\end{aligned}
$$

corresponding to $\check{a} \cdot \mathrm{y}+\check{c} \cdot \widetilde{\mathrm{y}}=\mathrm{A}\left(f^{-}, f_{+}\right)$, we obtain $\check{a} \cdot \mathrm{y}+\check{c} \cdot \widetilde{\mathrm{y}}=\hat{\mathrm{a}}\left(\varphi^{-}, \varphi_{+}\right)$, where the components

$$
\begin{aligned}
& \varphi^{-}(t)=(\check{\sigma} * \widetilde{\xi})(-t)+(\check{\sigma} * \eta)(t)=\widehat{f}^{-}(t) \\
& \varphi_{+}(t)=(\check{\sigma} * \xi)(t)+(\hat{\sigma} * \eta)(-t)=\widehat{f}_{+}(t)
\end{aligned}
$$

are given by the convolutions

$$
(\check{\sigma} * \zeta)(t)=\int_{-\infty}^{\infty} \check{\sigma}(t-r) \zeta(r) \mathrm{d} r
$$

with transition function

$$
\check{\sigma}(t)=\int \exp \{2 \pi \mathrm{i} \nu t\} \sigma(\nu) \mathrm{d} \nu=\hat{\sigma}(-t)
$$

Thus, the generalized processes y and $\widetilde{y}$ are obtained by the stationary filters

$$
\begin{aligned}
& \mathrm{y}(t)=\int_{-\infty}^{\infty} \check{\sigma}(s-t) \hat{\mathrm{A}}_{-}(\mathrm{d} s)+\int_{-\infty}^{\infty} \hat{\sigma}(s-t) \hat{\mathrm{A}}^{+}(\mathrm{d} s) \\
& \widetilde{\mathrm{y}}(t)=\int_{-\infty}^{\infty} \hat{\sigma}(s-t) \hat{\mathrm{A}}_{-}(\mathrm{d} s)+\int_{-\infty}^{\infty} \check{\sigma}(s-t) \hat{\mathrm{A}}^{+}(\mathrm{d} s)
\end{aligned}
$$

corresponding to the quantum stochastic integral representation

$$
\begin{aligned}
& \zeta \cdot \mathrm{y}=\int_{-\infty}^{\infty}(\check{\sigma} * \zeta)(s) \hat{\mathrm{A}}_{-}(\mathrm{d} s)+\int_{-\infty}^{\infty}(\hat{\sigma} * \widetilde{\zeta})(s) \hat{\mathrm{A}}^{+}(\mathrm{d} s) \\
& \zeta \cdot \widetilde{\mathrm{y}}=\int_{-\infty}^{\infty}(\hat{\sigma} * \widetilde{\zeta})(s) \hat{\mathrm{A}}_{-}(\mathrm{d} s)+\int_{-\infty}^{\infty}(\check{\sigma} * \zeta)(s) \hat{\mathrm{A}}^{+}(\mathrm{d} s)
\end{aligned}
$$

for the test functions $\widetilde{\zeta}(t)=\zeta(-t)$ with the square integrable $\widehat{\zeta} \sigma$ and $\widehat{\zeta} \widetilde{\sigma}$.

\section{CONCLUSION}

Thus we proved that for each quantum stationary noise there exists a fundamental output process which replaces the noise if the time is reversed. The nondemolition observation of the quantum noise via the measurement of the fundamental output process provides the best mean square filtering of the noise and in the classical limit completely eliminates this noise.

The input and output stationary processes can be decomposed in the second order into an orthogonal pair, consisting of vacuum and thermal noises which are orthogonal to the vacuum and connected by a reversible input-output (modular) filter. The time-continuous representation of the stationary linear filters requires a second order theory of quantum stochastic integration.

The direct application of standard quantum stochastic integration with respect to the quantum white noise integrators is not possible for equilibrium (KMS) states because of the non-Markovian character of the corresponding standard quantum processes $\mathrm{x}$ and $\widetilde{\mathrm{x}}$.

But it is possible to modify this approach for the spectral domain where the stationary quantum processes are $\delta$-correlated. Using this approach in the second 
order, we proved that they can be canonically decomposed into the superpositions of the vacuum pair of direct and reversed noises and can be obtained by a linear filtering from the standard vacuum noises $\mathrm{x}$ and $\widetilde{\mathrm{x}}$.

In general the spectral quantum stochastic integrates need not to be adapted and homogeneous with respect to the frequency shifts and the treatment of quantum nonlinear filters and other transformations of quantum equilibrium noise requires non stationary and non adapted theory of quantum stochastic integration [10].

\section{Acknowledgment}

The first author (VPB) is grateful for the hospitality and support of the Tamagawa University where this paper was partially written.

\section{REFERENCES}

[1] Hudson R.L. and Parthasarathy K.R., Quantum Ito's formula and stochastic evolution, Commun. Math. Phys., 93, 301-323, 1984.

[2] Gardiner C.W., Quantum Noise, Springer-Verlag, 1991.

[3] Accardi L., Frigerio A., and Lu Y.G., An invitation to the weak coupling and low density limits, In Quantum probability and related topics VI, World Scientifics, Singapore, 3-61, 1991.

[4] Belavkin V.P. Quantum Filtering of Markov Signals with White Quantum Noise, Radiotechnika and Electronika, 25, 1445-1453, 1980. The translation from Russian is published in this volume.

[5] Collett M.J. and Gardinar C.W., Input and output in damped quantum system: quantum stochastic differential equation and master equation, Phys. Rev. A 31, 3761-3774, 1985.

[6] Belavkin V.P., A quantum stochastic calculus in Fock space of input and output nondemolition processes, Springer-Verlag LNM 1442, 99-125, 1990.

[7] Parthasarathy K.R., An introduction to quantum stochastic calculus, Birkhausen, 1992.

[8] Hudson R.L. and Lindsay J.M., A noncommutative martingale representation for non-Fock quantum Brownian motion, J. Funct. Anal., 61, 202-221, 1985.

[9] Belavkin V.P., A quantum nonadapted Itô formula and stochastic analysis in Fock scale, J. Funct. Anal., 102, 414-447, 1991.

[10] Belavkin V.P., Chaotic states and stochastic integration in quantum systems, Russian Mathematical Survey, 1, 1-50, 1992.

[11] Lindsay M., Noncausal stochastic integration, Probability Theory and Related Topics, 1993.

[12] Belavkin V.P., Continuous non-demolition observation, quantum filtering and optimal estimation, In: Quantum Aspects of Optical Communication, Springer-Verlag, LNP378, 151-163, 1991.

[13] Belavkin V.P., Nondemolition principle of quantum measurement theory, Foundation of Physics, 1994.

[14] Belavkin V.P., Optimal linear randomized filtration of quantum boson signals, Problems of Control and Information Theory, 3(1), 47-62, 1974.

(A. One and A. Three) Mathematics Department, University of Nottingham, UK E-mail address: vpb@maths.nott.ac.uk

$U R L:$ http://www.maths.nott.ac.uk/personal/vpb/

Research Center of Quantum Communications, Tamagawa University, Tokyo, Japan 